\title{
Fundamentos humanos e históricos de la nutrición clínica
}

\author{
Human and historical foundations of clinical nutrition \\ Fundamentos humanos e históricos da nutrição clínica
}

\author{
Mónica López Talavera ${ }^{1 *}$
}

Recibido: 10 de junio de 2020. Aceptado para publicación: 5 de agosto de 2020

Publicado primero en línea: 21 de agosto de 2020

https://doi.org/10.35454/rncm.v3n2.160

\section{Resumen}

El quehacer de la nutrición clínica como disciplina científica que apoya la medicina y otras ciencias de la salud, tiene que reflexionarse desde sus antecedentes históricos para tener mayor claridad respecto a su propósito y el deber ser de los profesionales. La nutrición moderna era caracterizada como una ciencia fundamentada en la bioquimíca hasta el siglo XX cuando se comienza a reconocer que la especialización no es suficiente y que se requiere considerar la complejidad de los patrones alimentarios. Estos están determinados por la persona y el entorno en el que se desarrolla, por lo que diseñar una intervención nutricional significa conocer al paciente para implementar una terapéutica que le ayude a mejorar su vida desarrollar el cuidado y responsabilidad mediante la educación, tal como lo buscaban los terapeutas dietéticos de la antigüedad.

Por su parte, la nutrición clínica, que se entiende como nutrición artificial por sus antecedentes históricos los cuales se encuentran en el desarrollo de la medicina quirúrgica, con el alto grado de especialización muchas veces ha dejado de lado la complejidad, misma que debería retomarse tal como los primeros dietoterapeutas, para ser una disciplina clínica por su fundamento humano que no se limita a un espacio clínico.

Palabras clave: ciencias nutricionales, historia, ética, dietoterapia.

\section{Summary}

To improve our understanding on the purpose of clinical nutrition as a scientific discipline that supports medicine and other health sciences, and the professional obligation of clinicians towards it, we must ponder it from a historical background. Modern nutrition had been regarded as a science grounded on biochemistry until the 20th century, when it starts to be acknowledged that specialization is not enough and requires considering the complexity of eating patterns. These are determined by the person and the environment in which he or she develops. Therefore, designing a nutritional intervention involves having enough information on the patient to implement a therapy that helps improve their lives by promoting care and responsibility through education, just as the former dietary therapists did.

Historically, clinical nutrition, has been regarded as artificial nutrition due to its historical roots, which are found in the medical specialty surgery. Consequently, the high degree of specialization has often left aside the complexity of clinical nutrition, which should be regained to become a clinical discipline as practiced by the ancient diet therapists due to its human basis that is not limited to a clinical setting.

Keywords: Nutritional sciences; History; Ethics; Dietetics.

\section{Resumo}

A tarefa da nutrição clínica como disciplina científica que apoia a medicina e outras ciências da saúde deve refletir desde os seus antecedentes históricos para ter uma maior claridade em relação aos seus propósitos e a razão se ser dos seus profissionais. A nutrição moderna foi caracterizada como uma ciência baseada na bioquímica, no século XX começa a se reconhecer que a especialização não é suficiente e requer contemplar a complexidade dos padrões alimentares. Eles são determinados pela pessoa e pelo meio em que se desenvolve; portanto, projetar uma intervenção nutricional significa conhecer o paciente para implementar uma terapia que o ajude a melhorar sua vida, a desenvolver cuidados e responsabilidades por meio da educação, tal como buscavam os terapeutas dietéticos na antiguidade.

Por outro lado, a nutrição clínica, que é entendida como nutrição artificial devido aos seus antecedentes históricos encontrados no desenvolvimento da medicina cirúrgica, com o alto grau de especialização muitas vezes deixou de lado a complexidade, que deve ser retomada como os primeiros dietoterapeutas, para ser uma disciplina clínica devido ao seu fundamento humano que não se limita a um espaço clínico.

Palavras-chave: ciências nutricionais, história, ética, dietoterapia.

\footnotetext{
Universidad Autónoma del Estado de México, México
} 


\section{INTRODUCCIÓN}

Para que la nutrición clínica pueda ser comprendida a mayor profundidad, es necesario que se haga un recorrido, aunque breve, de la historia de su surgimiento, porque la manera como es concebida en la actualidad, es el resultado de la conjunción de saberes y disciplinas que se han generado en tres principales áreas del conocimiento a lo largo del tiempo como consecuencia del desarrollo técnico, científico y tecnológico.

Es por esto que en este escrito se intentará aclarar un poco más lo que se entiende como nutrición clínica, pero desde sus fundamentos histórico y humanístico. De esta manera se tendrá un recorrido por tres apartados en los que se hablará de la nutrición moderna, la dietética de la antigüedad y la clínica como ejercicio y espacio, que al fusionarse, dan como resultado la nutrición clínica.

La nutrición moderna es la que genera el conocimiento bioquímico y fisiológico de la interacción de los alimentos y sus efectos en el organismo; por su parte, la dietética, además de enfocarse en la alimentación, es la que nos marca la importancia del análisis del estilo de vida y sus repercusiones en la salud; y la clínica, es la que recuerda que el ejercicio de la nutrición clínica, al conjuntar efecto de los alimentos, conocimiento del ser humano y su estilo de vida, únicamente puede darse con la interacción entre dos o más personas, sin importar si se cuenta con el espacio clínico, pues con el acto es suficiente.

De esta forma, hablar de nutrición clínica es hablar de una disciplina que no debe enfocar su ejercicio únicamente en el ser humano desde una visión biológica, pues está sumergido en tal complejidad que obliga a retomar los conocimientos de la antigüedad que también consideran el modo de ser, las interacciones con el contexto y el trato humano.

\section{LA NUTRICIÓN MODERNA}

La nutrición moderna como disciplina científica comienza en el siglo XVIII durante la "revolución química” en la cual varios científicos reportaron estudios realizados con el método científico que permitieron comprender mejor el proceso nutritivo del ser humano ${ }^{(1)}$. Tal es el caso de los experimentos de Lavoisier, considerado "el padre de la nutrición", quien demuestra las bases del metabolismo y evidencia la existencia del oxígeno y la producción del dióxido de carbono y de agua en los tejidos animales ${ }^{(2)}$.

Es hasta mediados del siglo XIX que Justus von Liebig caracteriza la nutrición como ciencia bioquí- mica, permitiendo enfocar su desarrollo a partir del progreso científico de la época, en donde se buscaba la comprensión y manipulación de la naturaleza, específicamente del ser humano y su fisiología, mediante los alimentos y los efectos de los nutrientes específicos en el organismo. De esta manera la nutrición delimitó su campo de estudio con la especialización provocando dificultades para relacionar sus hallazgos con la situación nutricional y de salud que están presentes en la actualidad por la complejidad que implica ${ }^{(3)}$.

Esta especialización de la nutrición es más clara a partir del siglo XX con investigaciones que fueron claves para la comprensión de los efectos de los nutrientes en la salud de la población, porque con ellas se pudieron identificar las vitaminas y las consecuencias de su deficiencia estableciendo las dosis diarias recomendadas; se detectaron las enfermedades crónicas y su relación con las grasas y azúcares; se crearon suplementos de vitaminas así como alimentos modificados en cuanto al aporte de nutrientes para contrarrestar las deficiencias específicas y prevenir las enfermedades crónicas; pero es hasta 1990 cuando comienza a tomarse en cuenta la complejidad que representa la relación entre salud, alimentación y nutrición ${ }^{(4)}$.

Este cambio de enfoque ha sido obligado pues con el tiempo ha existido un aumento en la prevalencia de enfermedades por carencia y por exceso; y una epidemia por las mismas causas, en donde la nutrición a pesar de su especialización no ha sido suficiente como herramienta de prevención ni como tratamiento para estos fenómenos patológicos ${ }^{(5)}$.

El desarrollo tecnológico y la especialización en el campo de la salud son inevitables; sin embargo, a mayor especialización mayor automatización y menor raciocinio para el ejercicio de la profesión, que en este caso aplicado a la nutrición, es riesgoso pues a partir del siglo XX las investigaciones han sido cada vez más específicas hasta llegar a un nivel genómico, pero al mismo tiempo han sido incapaces de proponer soluciones efectivas y accesibles que mejoren la salud de la población. De esta manera, aunque se produce evidencia científica para generar estrategias de prevención o tratamiento no ha sido suficiente; ya que se presenta la barrera de que la realidad es más compleja que la causalidad de un solo compuesto, en este caso del nutriente o alimento con una enfermedad específica ${ }^{(6)}$.

Tal es el caso de la creación y aplicación de las guías de práctica clínica de la nutrición basada en evidencia, las cuales son el resultado del desarrollo de la técnica y deben actuar como apoyo y potenciadores de la 
naturaleza curativa del ser humano ${ }^{(7)}$. Hasta ahora han beneficiado la optimización de recursos y la prescripción de tratamientos nutricionales de bajo riesgo para el paciente, pero para aplicar las recomendaciones de las mismas se debe reflexionar de lo que es adecuado de acuerdo con su entorno y evitar que se vea afectado. Para nutrir adecuadamente se debe conocer la realidad de la persona para ejercitar las facultades de juicio y reflexión porque tanto los nutrientes obtenidos como la salud dependen de las relaciones humanas con el contexto y del desarrollo personal, situación que tiene fundamentos hipocráticos.

I. Hoffmann (2003) retoma entre líneas esta visión de la antigüedad y propone que el objetivo de la nutrición como ciencia ha cambiado y ahora se enfoca en identificar patrones alimentarios adecuados para mantener la salud y prevenir la enfermedad. Es importante conocer los alimentos, sus compuestos y propiedades, pero el conocimiento debe complementarse con el fin de comprender el proceso de nutrición humana y brindar recomendaciones para usar la alimentación como herramienta la cual también depende del contexto. Por esto, la nutrición se enfrenta a problemáticas complejas que no pueden ser abordadas estrictamente por relaciones causales a partir de un solo componente ${ }^{(8)}$. De aquí que las ciencias humanas se puedan considerar apoyo y complemento de los estudios que se realizan en la actualidad para identificar la evolución de la historia y futuro de la aplicación de la disciplina nutricional, especialmente para reconocer la complejidad que permitirá tener una visión más completa de la nutrición humana ${ }^{(4)}$.

Como ejemplo se tiene la Declaración de Giessen donde se establece que los principios generales de la ciencia de la nutrición son éticos, porque para la complejidad que implica la nutrición y la situación actual, es necesaria la intervención de la filosofía para que se analice el papel de la responsabilidad y el cuidado como parte de la nutrición y su relación con el comportamiento alimentario, como reflejo de la forma de vida de la persona y mantenimiento de la salud ${ }^{(9)}$, tal como se hacía en la antigüedad.

\section{NUTRICIÓN Y DIETÉTICA}

El estudio de la nutrición estaba inmerso en la dietética. Los primeros registros de la relación entre la nutrición, alimentación y salud son africanos, aunque también se han encontrado egipcios, chinos e indios en los cuales se muestra la importancia de la alimentación y su relación con la salud, la enfermedad y como una herramienta que brinda el conocimiento y el entendimiento de desarrollarse a lo largo de la vida a nivel físico, emocional y mental; ya que el bienestar era sinónimo de salud, y contemplaba al ser humano junto con su contexto. Así la dietética era parte de una filosofía de vida ${ }^{(3)}$.

Este era el fundamento de la dietética griega, pues en sus inicios tomaba en cuenta la nutrición mediante la interacción de los alimentos con la composición del hombre y la situación de su entorno ${ }^{(10)}$, al mismo tiempo que veía la dieta apropiada como una condición para el bienestar corporal e intelectual ${ }^{(11)}$. Como surge de la medicina, Platón decía que la dietética era un arte porque más allá del enfoque materialista de la salud y la enfermedad que se pudiera tener, la dieta ejemplificaba el comportamiento moderado así como el desarrollo mental que permitía tener una vida en armonía, y en el caso de los enfermos ayudaba a fortalecer el cuerpo y el espíritu para su recuperación ${ }^{(11)}$.

Hipócrates es quien le da un enfoque precientífico por el método de estudio mediante la observación y la posiciona como parte de la terapéutica, la cual hace dos distinciones para su aplicación: es higiene para la prevención de enfermedades y mantenimiento de la salud, pero también es un tratamiento a partir del estudio minucioso de las propiedades nutritivas de los alimentos y su efecto en el desarrollo patológico para ayudar a que el ser humano volviera a su armonía natural combatiendo la enfermedad. Con él, igualmente, se considera un arte, principalmente por su aplicación para curación, mediante la prescripción de regímenes alimentarios específicos, porque su implementación dependía de la etapa de la enfermedad así como de su severidad, exigiendo un profundo conocimiento de la naturaleza humana y de la historia de la enfermedad, y la capacidad de juicio para actuar en el momento preciso a partir de la interpretación de lo observado ${ }^{(10)}$.

Era el tratamiento de primera línea en la medicina hipocrática, la cual se fundamentaba en el conocimiento de la naturaleza humana, el respeto hacia la misma, su capacidad de autorregulación y el cuidado que debe existir para el mantenimiento de la salud, buscando que, en caso de ser necesaria la recuperación o curación, se brinden soluciones naturales no invasivas mediante la modificación de la alimentación y lo que implica para que se realice como ayuda restablecedora de la armonía de la naturaleza humana ${ }^{(12)}$.

La dieta que es modificada con los patrones alimentarios reafirma su carácter moral y nutritivo al reflejar la forma de vida y la capacidad de moderar los apetitos mediante el desarrollo intelectual y el ejercicio de la 
responsabilidad ${ }^{(13)}$. Este era el objetivo de la dietoterapia de Galeno porque con su aplicación, además del restablecimiento de la salud, promovía que el paciente se reeducara para ser responsable de su forma de vida mediante la propia dieta, nutriendo el cuerpo y la mente específicamente con su alimentación, porque de ella depende que se mantenga la salud o se provoque la aparición de la enfermedad e igualmente brinda la disciplina para que se conduzca a lo largo de su vida ${ }^{(14)}$.

De este modo, al ser la dietética parte de la terapéutica, la cual es el conocimiento del tratamiento necesario para combatir enfermedades ${ }^{(7)}$, el dietoterapeuta es la primera persona a la que se dirigían porque estaba dedicado a trabajar para el otro con la finalidad de ayudarle en beneficio de la salud, viéndose obligado a establecer una relación con la otra persona y demostrando el ejercicio clínico.

\section{LA CLÍNICA Y NUTRICIÓN CLÍNICA}

La clínica ha tenido transformaciones de su concepción en el transcurrir del tiempo, incluso en el desacuerdo de su surgimiento. Según Foucault la clínica existía mucho antes de que fuera el campo práctico de la enseñanza de la medicina o un espacio físico para atender al paciente porque surge de la relación del ser humano con su colectivo y con él mismo. Esto porque a lo largo de su existencia vive experiencias que le pueden causar sufrimiento físico, emocional o mental y que buscan remediarse como una necesidad para volver a sentirse bien ${ }^{(15)}$.

Con el progreso de la humanidad y la tecnología, la clínica se fue sistematizando en cuanto a su manera de practicarse y de enseñarse sin dejar de lado que implica un autoconocimiento de la naturaleza del hombre. Los estudiosos de la clínica obtenían su aprendizaje en el espacio mismo del enfermo, es decir, directamente acompañándole en su lecho. En el siglo XVIII comenzó a organizarse la enseñanza clínica en instituciones como las casas de salud y hospitales que tenían camas para atender enfermos en donde los más experimentados daban cátedra a los que querían dedicarse al arte de curar ${ }^{(15)}$.

Esta organización institucional a finales de siglo XVIII y principios del XIX ocasiona que la clínica se afirme como parte esencial de la enseñanza de la salud, conjuntando práctica y teoría, para brindar el servicio que proporcione beneficios a la humanidad, pues implica el estudio de su naturaleza así como su desarrollo dentro del contexto a partir del análisis de la enfermedad y su origen directamente en la cama del paciente $^{(15)}$.

Esta clínica, reconocida como clásica, en esencia es la interacción terapeuta - paciente, en donde el primero es el servidor que cumple de manera paternalista las demandas del paciente, y el segundo debe, después de solicitar el servicio para exponer sus malestares y sin opinión válida para la decisión final, acatar las órdenes del terapeuta, quien es responsable de recabar la información necesaria e interpretarla para prescribir una terapéutica de la que es responsable y, el límite de su intervención, deja al paciente la responsabilidad de llevar a cabo la prescripción ${ }^{(16)}$.

En la actualidad, la clínica depende de la tecnología y actúa basada en la evidencia. Al mismo tiempo, ocurre que el paciente tiene un papel activo, busca información y modifica la interacción que tiene con el terapeuta. Lo interesante es que, por esta misma actividad, el terapeuta encamina sus acciones y la prescripción de la terapéutica a la educación, necesaria para que el paciente sea responsable de su autocuidado. Desde este punto de vista la práctica ha dejado de ser paternalista pues los dos actores interactúan como seres humanos, haciendo que sea una experiencia y un ámbito sumergido en la complejidad porque existen influencias tecnológicas, informáticas, económicas, sociales e incluso institucionales que determinan las acciones clínicas: el análisis, el diagnóstico y el tratamiento ${ }^{(16)}$.

Además, la clínica se enfrenta a la situación de que su ejercicio cotidiano de manera ambulatoria va en aumento por las modificaciones epidemiológicas en las que prevalecen las enfermedades crónicas sobre las agudas, es decir que su aplicación y desarrollo en la cama del paciente como práctica cotidiana va a la baja. Aun así, su esencia de estudiar al paciente con padecimientos específicos en la cama (que en este caso sería en el hospital), está vigente y, sin importar si es aplicada de una u otra manera, continúa siendo una actividad con fundamento en las humanidades porque existe gracias a la interacción humana con propósitos fundamentalmente humanitarios ${ }^{(16)}$, lo cual también aplica a la nutrición desde sus inicios.

La nutrición clínica, entendida como la alimentación artificial, comenzó hace 5.000 años con los egipcios pues alimentaban aquellos pacientes que no podían ingerir alimentos por vía oral, por medio de enemas al igual que en la India, China e incluso en la antigua Grecia; ya que se comenzaba a observar que los alimentos tenían un efecto especial en el organismo 
tanto de las personas sanas como de las que padecían alguna enfermedad. Respecto a la alimentación endovenosa, el primer registro encontrado es del siglo XII, pero es a finales del XVI y principios del XVII cuando se impulsa el desarrollo de la alimentación enteral y parenteral paralelo al desarrollo de los espacios clínicos para el tratamiento de enfermedades, situación que prevalece hasta la actualidad pues se ha acelerado con el avance tecnológico y científico que se tiene en el siglo $\mathrm{XX}^{(17)}$ como se expuso en párrafos anteriores, llegando a transformarse en una disciplina basada en evidencias científicas para la sobrevivencia del ser humano.

Dentro del nacimiento de los espacios clínicos destinados a la atención de enfermedades en el siglo XVIII, el cuidado nutricional ya estaba como parte de la práctica clínica pues en los hospitales se daba de comer como acto de beneficencia para quien debía estar convaleciente. Posteriormente, en el siglo XIX, con la especialización de la práctica médica, la alimentación se delegó a los organismos administrativos de las instituciones los cuales observaron y reconocieron que la atención nutricional y la alimentación podían tener impacto económico, pero la terapia nutricional era escasa y no se realizaba ningún tipo de investigación al respecto.

Fue hasta el siglo XX cuando comenzó la búsqueda de alternativas para alimentar a las personas heridas en guerra a pesar de los procedimientos médicos quirúrgicos, pues comenzaron a observar que la alimentación podía cambiar completamente el curso de la recuperación. De aquí comenzó la investigación de la nutrición en el área clínica, porque la alimentación y la nutrición dejaron de verse solamente como una estrategia económica, política y social, para transformarse en determinantes para la sobrevivencia, complicaciones y mortalidad de las personas hospitalizadas ${ }^{(18)}$.

Florence Nightingale es una de las pioneras en reconocer a la alimentación como parte del tratamiento para los pacientes hospitalizados y a sus alumnos los alentaba a adentrarse en el estudio de la nutrición para poder aplicarla en el ámbito clínico, pero además estaba consciente de que prescribir la alimentación no era seguir la receta de un libro pues debía ser individualizada ${ }^{(17)}$ y al mismo tiempo permitía una práctica humanizada ya que, además, es parte de los cuidados que se deben brindar en el hospital. Hasta aquí, puede verse como un ejercicio en el espacio clínico. Pero al mismo tiempo es un acto clínico porque se habla de una individualización porque cada ser humano es diferente así como su concepción de la vida y del contexto en el que se ha formado y, por lo mismo, establecer una relación directa entre el dietoterapeuta y el paciente es obligado dando el tinte de clínico a la nutrición aplicada como terapéutica.

\section{CONCLUSIÓN}

Concebir la nutrición clínica únicamente como alimentación artificial limita su campo de acción. La clínica junto con la nutrición humana no se circunscribe al acto de alimentar, también es cuidado y educación, tanto para el paciente como para quienes lo rodean, con el fin de modificar su dieta o modo de vida y ayudar en la curación o mantenimiento de la salud. Aunque su desarrollo se ha reconocido por el gran avance técnico y tecnológico respecto a sus procedimientos y herramientas, en los cambios y variaciones para dar soluciones más especializadas con el estudio de los procesos fisiológicos y necesidades nutricionales del cuerpo físico del ser humano que han permitido disminuir la mortalidad y complicaciones, así como los costos y mejorar la optimización de recursos tanto humano como materiales, aún se tienen dos grandes problemas de malnutrición que se han detectado a partir del siglo $\mathrm{XX}$ : la desnutrición hospitalaria y la obesidad, ambas como causantes de mayores complicaciones de salud. Estas situaciones pueden sugerir que la especialización no es suficiente para mejorar el cuidado y la educación respecto a la nutrición adecuada.

Para estos perfiles patológicos, la nutrición clínica debe retomar el fundamento que se encuentra en la relación humana entre dos actores o más para brindar una terapéutica efectiva. Por lo tanto, la nutrición clínica debe buscar nutrir al ser humano de manera integral y al realizar ese cambio en la dieta, que también es entendida como modo o forma de vida. Podría llamarse entonces, dietoterapia clínica pues el enfoque del profesional y su intervención estarán dirigidas a la formación de hábitos saludables con recomendaciones personalizadas teniendo en cuenta la necesidad de conocer de la mejor manera posible al paciente.

\section{Declaración de conflicto de intereses}

Ninguno.

\section{Financiación}

Consejo Nacional de Ciencia y Tecnología, CONACyT. 


\section{Referencias bibliográficas}

1. Carpenter KJ. A short history of nutritional science: Part 1 (1785-1885). J. Nutr. 2003;133(3):638-45. DOI https://doi. org/10.1093/jn/133.3.638.

2. Bascunán BA. Antoine Laurent Lavoisier. El revolucionario. Educación Química. 2008;19(3):226-33. DOI http://dx.doi. $\mathrm{org} / 10.22201 /$ fq.18708404e.2008.3.25836.

3. Cannon $\mathrm{G}$. The rise and fall of dietetics and nutrition science, 4000 BCE-2000 CE. Public Health Nutr. 2005;8(6a):701-5. DOI https://doi.org/10.1079/phn2005766.

4. Mozaffarian D, Rosenberg I, Uauy R. History of modern nutrition science - implications for current research, dietary guidelines, and food policy. BMJ. 2018;361:K2392. DOI https://doi.org/10.1136/bmj.k2392.

5. Fardet A, Rock E. Toward a new philosophy of preventive nutrition: from a reductionist to a holistic paradigm to improve nutritional recommendations. Adv. Nutr. 2014;5(4):430-46. DOI https://doi.org/10.3945/an.114.006122.

6. Jaspers K. La práctica médica en la era tecnológica. España:Gedisa; 2003.

7. GadamerHG.Elestadooculto delasalud.España:Gedisa;1996.

8. Hoffmann I. Transcending reductionism in nutrition research. Am J Clin Nutr. 2003; 78(3):514S-6S. DOI https://doi. org/10.1093/ajcn/78.3.514S.

9. Beauman C, Cannon G, Elmadfa I, Glasauer P, Hoffmann I, Keller M, et. al. The Giessen Declaration. Public Health Nutr. 2005;8(6a):783-6. DOI https://doi.org/10.1079/ PHN2005768.
10. Lonie IM. A structural pattern in Greek dietetics and the early history of Greek medicine. Med Hist. 1977;21(3):235-60. DOI https://doi.org/10.1017/s0025727300038242.

11. Skiadas P, Lascaratos J. Dietetics in ancient Greek philosophy: Plato's concepts of healthy diet. Eur J Clin Nutr. 2001;55:5327. DOI https://doi.org/10.1038/sj.ejcn.1601179.

12. Alby JC. La concepción antropológica de la medicina hipocrática. Enfoques XVI. 2004;16(1):5-29. DOI https://doi. org/10.17843/rpmesp.2017.342.2988.

13. Medina González LM. Dietética y Moral. Medicina y filosofía en la antigüedad helenística. Estud. Filos. 2010; 42:209-50.

14. Cacciafoco FP. Food as Therapy. Elements of the History of Nutrition in Ancient Greece and Rome. Pollenzo (IT): International University of Pollenzo - UNISG: Teatro Vocali Edizioni; 2012.

15. Foucault M. El nacimiento de la clínica: una arqueología de la mirada médica. México: Siglo XXI editores; 2015.

16. Lifshitz A. La nueva clínica. México: Intersistemas S. A. de C. V.; 2014.

17. Vassilyadi F, Panteliadou AK, Panteliadis C. Hallmarks in the history of enteral and parenteral nutrition: from antiquity to the 20th century. Nutr Clin Pract. 2013;28(2):209-17. DOI https://doi.org/10.1177/0884533612468602.

18. Cardenas D. What is clinical nutrition? Understanding the epistemological foundations of a new discipline. Clin Nutr ESPEN. 2016;11:e64. DOI https://doi.org/10.1016/j. clnesp.2015.10.001. 